\title{
TỔNG HỢP XANH NANO VÀNG BẦNG DỊCH CHIẾT VỎ TRÁI THƠM VÀ ÚNG DỤNG LÀM XÚC TÁC CHO PHẢN ÚNG XỦ LÝ 4-NITROPHENOL
}

\author{
NGUYẼ̃N THI LAN HƯƠNG \\ Viện Công nghệ Sinh học \& Thục phẩm, Trương Đại học Công nghiệp Thành phố Hồ Chi Minh \\ nguyenthilanhuong@iuh.edu.vn
}

Tóm tắt. Trong công trình này, nano vàng (AuNPs) đã được tổng hợp thành công bằng phương pháp một bước đơn giản sử dụng chiết xuất từ vỏ trái thơm $(\mathrm{PP})$, một nguồn phế thải nông nghiệp để làm chất khử cũng như chất ổn định. Các thông số chính ảnh hưởng đến tổng hợp PP-AuNPs, bao gồm nồng độ ion vàng, thời gian phản ứng và nhiệt độ phản ứng được tối ưu hóa bằng quang phổ hấp thụ tử ngoại - khả kiến (UVVis) với độ hấp thụ cực đại đặc trưng cho nano vàng tại $560 \mathrm{~nm}$. Các nhóm chức hữu cơ đóng vai trò khử các ion vàng và ổn định các hạt $\mathrm{PP}$ AuNPs được kiểm tra bằng quang phổ hồng ngoại biến đổi Fourier (FTIR). Phân tích nhiễu xạ tia X (XRD) đã xác định cấu trúc tinh thể của PP_AuNPs. Các phép đo trên kính hiển vi điện tử truyền qua (TEM) cho thấy các hạt PP_AuNPs chủ yếu có dạng hình cầu với kích thước nhỏ trong khoảng 5-15 nm. Các hạt nano vàng cũng đã thể hiện hoạt tính xúc tác khá tốt trong việc khử hoàn toàn 4-nitrophenol thành 4-aminophenol trong 10 phút với hằng số tốc độ phản ứng là 0,233 phút ${ }^{-1}$.

Từ khóa. Nano vàng, tổng hợp xanh, vỏ trái thơm, hoạt tính xúc tác, phản ứng khử 4-nitrophenol.

\section{GREEN SYNTHESIS OF GOLD NANOPARTICLES BASED ON EXTRACT FROM AGRICULTURAL WASTE OF PINEAPPLE PEELS FOR CATALYTIC REDUCTION TOWARD 4-NITROPHENOL}

\begin{abstract}
Biogenic gold nanoparticles (AuNPs) were successfully synthesized by a simple single-step method using extract from agricultural waste of pineapple peels (PP) as reducing as well as stabilizing agent. Major parameters affecting the formation of PP-AuNPs, including gold ions concentration, reaction time, and reaction temperature were optimized using ultraviolet-visible (UV-Vis) measurements at characteristic maximum absorbance of $560 \mathrm{~nm}$. The functional groups responsible for reducing gold ions and capping AuNPs were examined by Fourier-transform infrared (FTIR) spectroscopy. Powder X-ray diffraction (XRD) analysis confirmed the crystalline nature of PP_AuNPs. Transmission electron microscopy (TEM) measurements showed that the biosynthesized PP-AuNPs were mostly spherical with an average size of 5-15 nm. The gold nanoparticles also demonstrated excellent catalytic activity for the complete reduction of 4-nitrophenol to 4-aminophenol in the presence of $\mathrm{NaBH}_{4}$ within 10 min with reaction rate constant of $0.233 \mathrm{~min}^{-1}$.
\end{abstract}

Keywords. Gold nanoparticles, biosynthesis, pineapple peel, catalytic activity, 4-nitrophenol reduction.

\section{GIỚI THIỆU}

Trong những năm gần đây, lĩnh vực công nghệ nano ngày càng thu hút sự quan tâm nghiên cứu của các nhà khoa học. Trong những loại vật liệu nano đầy triển vọng, không thể không nhắc đến các hạt nano kim loại quý, đặc biệt là nano vàng (AuNPs), vì chúng được sử dụng rất sớm và có rất nhiều ứng dụng trong trong xúc tác, kháng khuẩn, kháng nấm, và cảm biến sinh học [1-4].

Để kiểm soát kích thước và hình thái của các hạt nano vàng, nhiều phương pháp tổng hợp từ vật lý đến hóa học đã được đề xuất, điển hình là các phương pháp khử hóa học sử dụng hydrazine, sodium borohydride, acid ascorbic, glycerol nhằm chuyển hóa ion vàng thành vàng kim loại ở dạng kích thước nano. Uu điểm của những phương pháp này là thời gian tiến hành tổng hợp nhanh và dễ dàng nhưng có hạn chế lớn là độc tính từ hóa chất sử dụng làm chất khử có thể ảnh hưởng đến phạm vi ứng dụng của sản phẩm nano và việc loại bỏ chúng là rất khó khăn [5]. Ngày nay, phương pháp tổng hợp xanh dùng dịch chiết của thực vật làm tác nhân khữ đã nhận được sự quan tâm rất lớn tử các nhà nghiên cứu vì phương pháp tổng hợp này rất hiệu 
quả, tiềm năng sản xuất công nghiệp với chi phí thấp thấp, nhưng cho hoạt tính sinh học cao, tận dụng các nguyên liệu từ tự nhiên, quy trình tổng hợp đơn giản và thân thiện với môi trường [6]. Nhiều công trình nghiên cứu báo cáo về tổng hợp nano vàng sử dụng dịch chiết thực vật, như Lactuca indica, Fritillaria cirrhosa [7], Jasminum auriculatum leaf [8], Commiphora wightii [9], red cabbage [10], Flammulina velutipes [11], Euphorbia fischeriana root [12], Solanum nigurum [13].

Nhiều nhà nghiên cứu báo cáo rằng AuNPs thể hiện hoạt động xúc tác mạnh mẽ trong phản ứng hydro hóa các hợp chất nitrophenol độc hại thành các chất không gây hại aminophenols tương ứng $[14,15]$. Trong số đó, 4-nitrophenol (4-NP) thải ra chủ yếu từ các hoạt động sản xuất phân bón, hóa dầu và thuốc nhuộm được coi là một chất ô nhiễm hữu cơ nguy hiểm trong nước thải công nghiệp [16]. Phản ứng khử 4-NP bằng dung dịch $\mathrm{NaBH}_{4}$ sử dụng AuNPs trong vai trò của chất xúc tác đã được nghiên cứu rộng rãi $[17,18]$. Cần chú ý rằng, phản ứng khử nitrophenol với $\mathrm{NaBH}_{4}$ khi không có chất xúc tác thuận lợi về mặt nhiệt động, nhưng không thuận lợi về mặt động học do năng lượng hoạt hóa lớn. Sự hiện diện của các hạt nano kim loại, đặc biệt là AuNPs, đã làm năng lượng hoạt hóa của phản ứng giảm xuống thông qua cơ chế chuyển điện tử của chất xúc tác, dẫn đến phản ứng xảy ra với tốc độ nhanh hơn. Ở đây, cả hai ion borohydride và nitrophenolate đóng vai trò là chất cho và nhận điện tử trên bề mặt chất xúc tác [19].

Thực tế cho thấy, chế biến thực phẩm sẽ luôn tạo ra một lượng chất thải nhất định. Ước tính hàng năm, tổng giá trị thiệt hại thực phẩm đối với ba dòng chất thải nông nghiệp lớn bao gồm ngũ cốc (36,1 tỷ USD), rau (108,7 tỷ USD) và trái cây (62,2 tỷ USD) [20]. Vì thế, đã có nhiều công trình khoa học nghiên cứu về việc ứng dụng các nguồn phế thải nồng nghiệp này để tổng hợp các hạt nano kim loại mang lại giá trị cao [21]. Nước ép từ phần thịt trái thơm đã được sử dụng để tổng hợp nano vàng cho ứng dụng kháng khuẩn [22], nhưng theo sự hiểu biết của chúng tôi, hiện nay vẫn chưa có công trình khoa học nào công bố việc sử dụng nguồn phế thải vỏ trái thơm (pineapple peel, $\mathrm{PP}$ ) để tổng hợp AuNPs cho các ứng dụng xúc tác. Trong công trình này, chúng tôi tiến hành nghiên cứu tổng hợp nano vàng từ dung dịch $\mathrm{HAuCl}_{4} .3 \mathrm{H}_{2} \mathrm{O}$ với tác nhân khử là dịch chiết từ vỏ trái thơm, một nguồn phế thải nông nghiệp và đánh giá hoạt tính xúc tác của AuNPs trong phản ứng khử 4-NP bằng dung dịch $\mathrm{NaBH}_{4}$.

\section{NGUYÊN LIỆU VÀ PHƯƠNG PHÁP NGHIÊN CỨU}

\subsection{Nguyên liệu}

Hydrogen tetrachloroaurate (III) hydrat $\left(\mathrm{HAuCl}_{4} \cdot 3 \mathrm{H}_{2} \mathrm{O}\right)$, sodium tetrahydridoborate $\left(\mathrm{NaBH}_{4}\right)$ được mua từ công ty hóa chất Acros (Belgium). 4-nitrophenol $\left(\mathrm{C}_{6} \mathrm{H}_{5} \mathrm{NO}_{3}\right)$ được cung cấp bởi công ty Merck (Ấn Độ). Tất cả dụng cụ thủy tinh được tráng rửa sạch sẽ bằng nước cất trước khi sử dụng để tránh hiện tượng keo tụ dung dịch nano vàng gây ra bởi các chất điện ly.

\section{2 Điều chế dịch chiết từ vỏ trái thơm}

Vỏ trái thơm được thu lượm tại khu vực bán trái cây ở chợ Gò Vấp, TP. HCM. Vỏ được rửa sạch để loại bỏ hoàn toàn chất bẩn, sấy khồ và cắt thành các mảnh nhỏ. Cân khoảng $100 \mathrm{~g}$ vỏ trái thơm cắt nhỏ cho vào $200 \mathrm{~mL}$ nước cất, đun sôi hoàn lưu trong thời gian 1 giờ. Hỗn hợp sau đun được làm nguội tới nhiệt độ phòng, lọc bằng giấy lọc Whatman No. 1 để loại bỏ chất rắn hữu cơ không tan. Phần dịch chiết được bảo quản trong ngăn mát tủ lạnh ở $4-8{ }^{\circ} \mathrm{C}$ chuẩn bị cho các thí nghiệm tiếp theo.

\subsection{Tổng hợp PP_AuNPs}

Quá trình tổng hợp nano vàng được thực hiện với dung dịch $\mathrm{HauCl}_{4}$ và dịch chiết vỏ trái thơm theo tỉ lệ 1:20 theo sơ đồ Hình 1. Sự thay đổi màu của dung dịch sau phản ứng từ màu vàng nhạt của dịch chiết sang màu tím đậm chứng tỏ nano vàng đã được tổng hợp thành công. Các yếu tố ảnh hưởng đến quá trình tổng hợp như nồng độ ion vàng $(0.5-2 \mathrm{mM})$, nhiệt độ $\left(60-90^{\circ} \mathrm{C}\right)$ và thời gian (45-90 phút) được khảo sát. Các yếu tố ảnh hưởng được tối ưu hóa thông qua phép đo UV-Vis với độ hấp thụ cực đại đặc trưng của nano vàng ở đỉnh $560 \mathrm{~nm}$. Nano vàng sau khi tổng hợp ở các điều kiện tối ưu được dùng để phân tích các đặc tính hóa lý và nghiên cứu ứng dụng của chúng. 


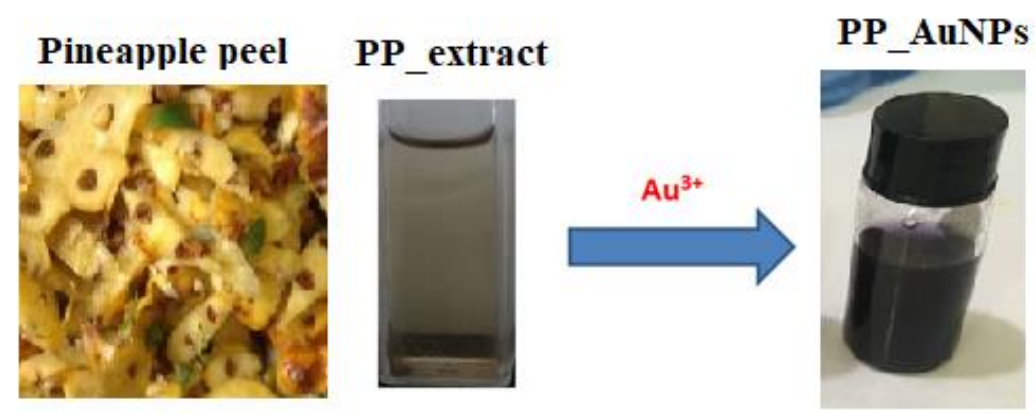

Hình 1: Sơ đồ tổng hợp PP_AuNPs từ dịch chiết vỏ trái thơm

\subsection{Phương pháp phân tích Xác định đặc tính hóa lý của PP_AuNPs}

Sự hiện diện của các nhóm chức có trong mẫu nano vàng và mẫu cao dịch chiết khô được phân tích bằng phương pháp phổ hồng ngoại biến đổi Fourie (FTIR) trên máy quang phổ 27 Brucker Tensor (Đức) trong phạm vi số sóng $4000-500 \mathrm{~cm}^{-1}$. Cấu trúc tinh thể của mẫu nano vàng được phân tích bằng phương pháp nhiễu xạ tia X trên máy Shimadzu 6100 diffractometer (Nhật Bản), hoạt động ở điện áp $40 \mathrm{kV}$, dòng điện $30 \mathrm{~mA}$ với bức xạ $\mathrm{CuK} \alpha$ có bước sóng $1,5406 \mathrm{~nm}$ với tốc độ quét $0,05 \%$, bước nhảy $0.02^{\circ}$ trong phạm vi $2 \theta$ từ $10^{\circ}$ đến $80^{\circ}$. Hình thái và kích thước của các hạt nano vàng được xác định bởi kính hiển vi điện tử truyền qua (TEM) và kính hiển vi điện tử quét (FE-SEM) trên máy JEOL JEM-2100 và máy FE-SEM $\mathrm{S}$ 4800 tương ứng. Phân bố kích thước hạt và thế zeta được đo bằng phương pháp động ọc phân tán ánh sáng (DLS) trên máy Horiba SZ-100 (Nhật Bản). Các tính chất nhiệt của hạt nano vàng được xác định bởi phương pháp phân tích nhiệt trọng lượng (TG) hết hợp phân tích nhiệt vi sai (DTA) sử dụng máy Evo LabSys $\mathrm{S} 60 / 58988$ (Pháp). Các mẫu khô được nung từ 30 đến $800^{\circ} \mathrm{C}$ với tốc độ gia nhiệt $10^{\circ} \mathrm{C} /$ phút trong điều kiện khí quyển.

\subsection{Hoạt tính xúc tác của AuNPs}

Khảo sát khả năng xúc tác của mẫu PP_AuNPs để loại bỏ các chất hữu cơ 4-NP bằng chất khử $\mathrm{NaBH}_{4}$ được thực hiện trong một cuvet tại nhiệt độ phòng. Dung dịch 4-NP $(2,5 \mathrm{~mL} ; 0,1 \mathrm{mM})$ được trộn với dung dịch $\mathrm{NaBH}_{4}(0,5 \mathrm{~mL} ; 0,1 \mathrm{M})$, sau đó thêm vào $3 \mathrm{mg}$ nano vàng để phản ứng diễn ra. Nano vàng sử dụng trong thí nghiệm này thu được bằng phương pháp ly tâm với tốc độ 8000 vòng/phút, sau đó loại bỏ nước rồi sấy khô. Động học của phản ứng xúc tác được đánh giá qua phép đo UV-Vis với sự suy giảm nhanh của 4-NP với độ hấp thụ cực đại ở $400 \mathrm{~nm}$. Để đơn giản hóa trong nghiên cứu về động học xúc tác, lượng $\mathrm{NaBH}_{4}$ được sử dụng vượt xa nồng độ chất gây ô nhiễm, nên nồng độ của $\mathrm{NaBH}_{4}$ thực tế được coi là không đổi trong suốt quá trình phản ứng. Do đó, phản ứng được coi là phản ứng bậc một giả kiến và được mô tả bởi phương trình $\ln (\mathrm{At})=\mathrm{f}(\mathrm{t})[15]$, trong đó $\mathrm{k}$ là hằng số tốc độ, $\mathrm{t}$ là thời gian phản ứng, và $[\mathrm{At}]$ là nồng độ chất ô nhiễm tại thời điểm khảo sát. Khi xây dựng đường hồi qui tuyến tính $\ln (\mathrm{At})$ theo thời gian sẽ thu được một đường thẳng và từ độ dốc của đường thẳng có thể xác định hằng số tốc độ phản ứng.

\section{KẾT QUẢ VÀ THẢO LUẬN}

\subsection{Tối ưu hóa tổng hợp PP_AuNPs}

Các thông số tổng hợp bao gồm nồng độ ion vàng, thời gian phản ứng và nhiệt độ phản ứng ảnh hưởng mạnh mẽ đến kích thước của các hạt nano vàng thu được. Quá trình tối ưu hoá các thông số này sẽ giúp thu được nano vàng có kích thước hợp lý, dung dịch có độ bền vững nhiệt động cao và khó bị keo tụ hơn theo thời gian. Trong công trình này, nồng độ ion vàng được khảo sát bằng cách thay đổi nồng độ dung dịch $\mathrm{HAuCl}_{4}$ trong khoảng $0,5-2 \mathrm{mM}$ trong khi các thông số nhiệt độ và thời gian, lần lượt là 90 phút và $90^{\circ} \mathrm{C}$ được giữ cố định (Hình 2(a)). Kết quả cho thấy nồng độ ion ảnh hưởng nhiều đến sự hình thành của nano vàng, nồng độ càng tăng độ hấp thụ quang học càng cao. Khi đến nồng độ nhất định nào đó thì độ hấp thụ bắt đầu giảm, điều đó cho thấy ở nồng độ cao các hạt nano vàng dễ bị keo tụ. Qua thực nghiệm xác định được nồng độ thích hợp của $\mathrm{HAuCl}_{4}$ để tổng hợp PP_AuNPs là $1,5 \mathrm{mM}$.

Bước kế tiếp, để tìm thời gian phù hợp cho phản ứng, dung dịch nano vàng cứ 15 phút được mang đi đo quang một lần, nồng độ và nhiệt độ được giữ cố định tại $1,5 \mathrm{mM}$ và $90^{\circ} \mathrm{C}$ (Hình $(2 \mathrm{~b})$ ). Kết quả quan sát ở 
Hình 2(b) cho thấy thời gian tối ưu để tổng hợp nano vàng là 60 phút. Với thời gian tổng hợp lớn hơn 60 phút thì độ hấp thụ quang học giảm cho thấy khả năng hạt hình thành to, dễ keo tụ.

Cuối cùng, nhiệt độ phản ứng được khảo sát trong khoảng $60-90^{\circ} \mathrm{C}$, sử dụng nồng độ và thời gian tổng hợp tương ứng là $1,5 \mathrm{mM}$ và 60 phút (Hình $2(\mathrm{c})$ ). Kết quả cho thấy nhiệt độ cũng ảnh hưởng rất lớn đến việc hình thành các hạt nano, nhiệt độ càng tăng thì độ hấp thụ quang học cũng tăng theo. Nhiệt độ tốt nhất để tổng hợp nano vàng là $80^{\circ} \mathrm{C}$. Ở nhiệt độ cao hơn, các phân tử chuyển động nhanh, số va chạm hiệu quả tăng nhanh dẫn đến hạt nano tạo thành nhanh, dễ bị keo tụ, hạt có kích thước lớn gây giảm mật độ quang.

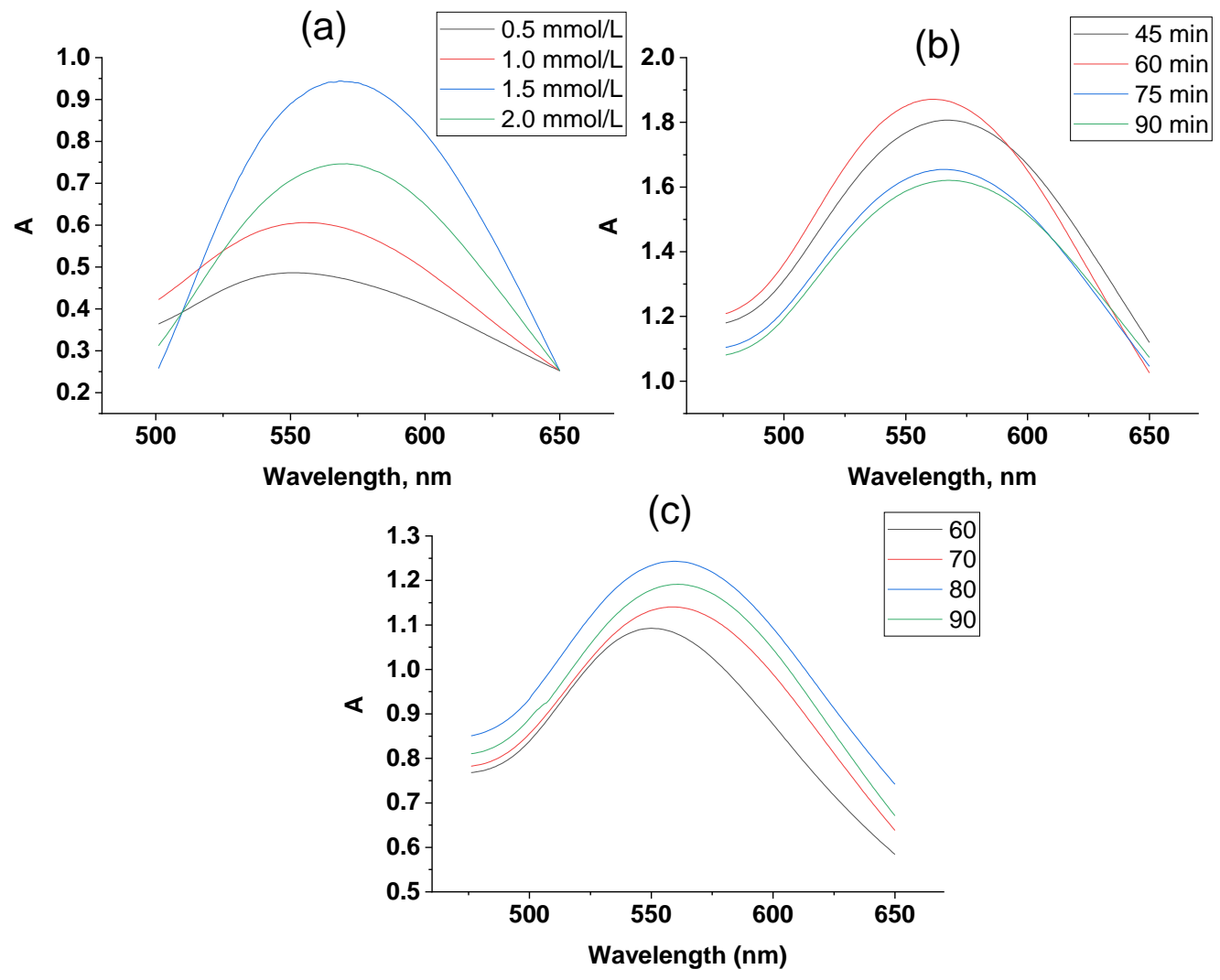

Hình 2: Phổ UV-Vis của dung dịch PP_AuNPs theo nồng độ (a), thời gian (b) và nhiệt độ (c)

\subsection{Tính chất hóa lý của PP_AuNPs}

\section{Kết quả phân tích nhiễu xạ tia $X$ và FT-IR}

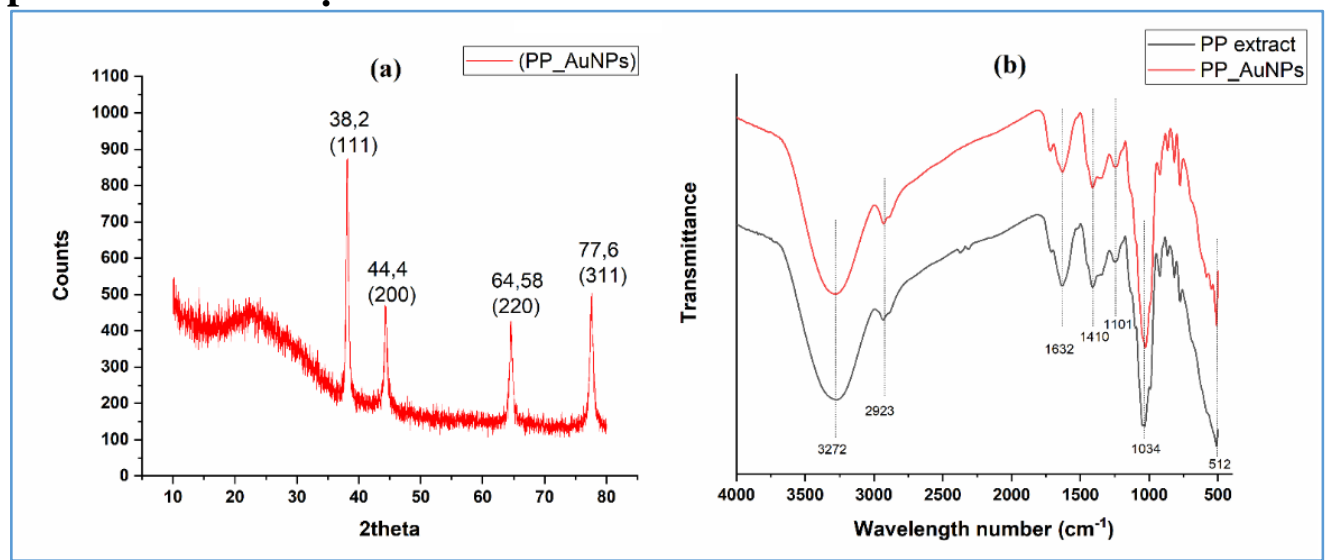

Hình 3: Giản đồ XRD và phổ FTIR của mẫu PP_AuNPs và cao dịch chiết 
Các đặc điểm cấu trúc tinh thể của PP_AuNPs được xác định bởi giản đồ XRD, trình bày trong Hình 3(a). Giản đồ XRD của mẫu PP_AuNPs hiện thị các peak ở các góc nhiễu xạ $2 \theta$ lần lượt là $38,12^{\circ}, 44,4^{\circ}, 64,58^{\circ}$ và $77,6^{\circ}$, đặc trưng cho các mặt phẳng (111), (200), (220) và (311) của tinh thể lập phương tâm diện $\mathrm{Au}$ tương ứng với giản đồ XRD chuẩn PDF ICDD 00-004-0784. Trong đó, đỉnh cao nhất là ở mặt (111), cho thấy các tinh thể phát triển ưu tiên theo mặt (111). Như vậy, khi xác định chiều rộng bán đỉnh ở mặt (111), có thể tính ra kích thước trung bình của tinh thể. Kích thước trung bình của tinh thể được xác định theo phương trình Debye-Scherrer $\mathrm{d}=0.9 \lambda / \beta \cos \theta$, với $\mathrm{d}$ là đường kính trung bình của tinh thể $(\mathrm{nm})$, ' $\beta$ ' là $(\pi / 180)$ x FWHM (full width at half maximum - chiều rộng bán đỉnh); ' $\lambda$ ' là nguồn bức xạ tia $X(0.1540$ $\mathrm{nm})$ and ' $\theta$ ' là góc Bragg. Theo đó, kích thước trung bình của tinh thể PP_AuNPs là 12,4 nm.

Kết quả phân tích FTIR của mẫu PP_AuNPs và mẫu cao dịch chiết sấy khô cho thấy sự xuất hiện các dải phổ ở các đỉnh 1034; 1101; 1410; 1632; 2923 và (Hình 3(b)). Các đỉnh phổ của nano vàng sau khi tổng hợp bằng dịch chiết hoàn toàn tương đồng với mẫu cao dịch chiết sấy khô. Đặc trưng cho dải phổ ở $3272 \mathrm{~cm}^{-1}$ là sự hiện diện của nhóm chức $-\mathrm{OH}$, chứng tỏ sự tồn tại của glucides, polyphenol và một số ít các phân tử nước [23]. Đỉnh phổ $1632 \mathrm{~cm}^{-1}$ tương ứng với liên kết $\mathrm{C}=\mathrm{O}$ của keton [4]. Đỉnh ở $1410 \mathrm{~cm}^{-1}$ có liên quan đến liên kết $\mathrm{C}=\mathrm{C}$ trong các hợp chất nhân thơm [24]. Các đỉnh ở 1101 và $1034 \mathrm{~cm}^{-1}$ đặc trưng cho liên kết $\mathrm{C}-\mathrm{O}$ của ether và nhóm chức axit cacboxylic, tương ứng [25]. Phổ FTIR cho thấy thành phần hữu cơ của dịch chiết vỏ trái thơm đã đóng vai trò như một chất làm bền hiệu quả cho hạt nano vàng.

\section{Thành phần nguyên tố của PP_AuNPs}

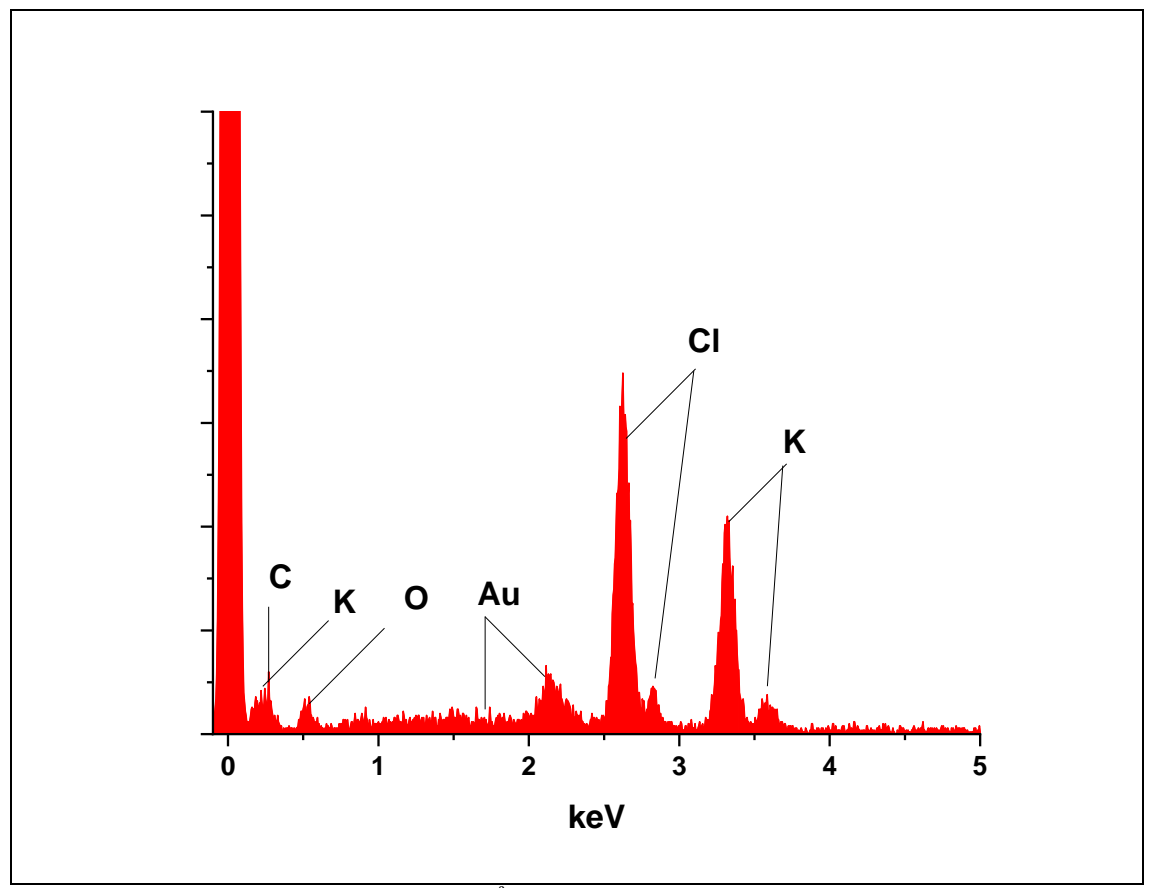

Hình 4: Phổ EDX của PP_AuNPs

Thành phần nguyên tố hóa học của mẫu $\mathrm{PP} \_$AuNPs được phân tích bằng phương pháp quang phổ tán sắc năng lượng tia $X$ (Hình 4). Để có thể đánh giá một cách tương đối chính xác sự hiện diện của các nguyên tố trong hợp chất hữu cơ cũng như vô cơ có trong dịch chiết vở trái thơm, mẫu PP_AuNPs sau tổng hợp được làm keo tụ, lọc và sấy khô mà không tách qua ly tâm. Phổ EDX chỉ ra rằng các hạt $\mathrm{PP} A \mathrm{AuNPs}$ chủ yếu bao gồm $\mathrm{Au}(8,41 \%)$ với các đỉnh đặc trưng ở 1,74 và $2,195 \mathrm{keV}$. Carbon $(11,05 \%)$ và oxy $(6,0 \%)$ cũng được quan sát ở đỉnh 0,255 và $0,53 \mathrm{keV}$, xác nhận sự hiện diện của các thành phần hữu cơ được phủ trên AuNPs. Ngoài ra, các nguyên tố vô cơ như K $(35,02 \%)$ và $\mathrm{Cl}(39,01 \%)$ cũng được tìm thấy. Sự hiện diện của các nguyên tố $\mathrm{K}$ và $\mathrm{Cl}$ trong nhiều chiết xuất thực vật đã được báo cáo trong một số công trình trước đây $[26,27]$.

\section{Hình thái và phân bố kích thước hạt}

Ảnh SEM, TEM của mẫu PP_AuNPs được thể hiện tương ứng trên Hình 5(a) và 5(b), cho thấy các hạt nano vàng có dạng hình cầu với kích thước hạt nhỏ trong khoảng 5-15 nm. Phân bố kích thước hạt trong dung 

VỎ TRÁI THƠM XÚC TÁC CHO PHẢN ÚNG XƯ LÝ 4-NITROPHENOL

dịch PP_AuNPS (Hình 4(c)) đã chỉ ra rằng, đường kính động học trung bình của hạt PP_AuNPs là 69,1 $\mathrm{nm}$, lớn hơn rất nhiều so với kích thước hạt chụp bằng phương pháp TEM. Sự khác biệt này chứng tỏ rằng các hạt PP_AuNPs đã được bao phủ bởi một lớp chất hữu cơ dày, giúp các hạt tránh bị keo tụ. Thế zeta của dung dịch $\mathrm{PP}$ AuNPs được xác định bằng $-18,2 \mathrm{mV}$ (Hình 4(c)). Như vậy, có thể kết luận rằng tồn tại song song hai cơ chế giúp bảo vệ các hạt PP_AuNPs.. Cơ chế thứ nhất dựa vào hiệu ứng không gian, lớp chất hữu cơ bao bọc bên ngoài hạt nano vàng đóng vai trò như lớp áo bảo vệ, giúp các hạt nano không dính lại với nhau. Cơ chế thứ hai dựa trên tương tác tĩnh điện giữa các hạt nano, cụ thể là các hạt PP_AuNPs cùng tích điện âm sẽ đẩy nhau, hạn chế hiện tượng keo tụ. Trên thực tế, nhờ cơ chế bảo vệ tích hợp này mà dung dịch PP_AuNPs sau khi tổng hợp có thể bền vững hơn một tuần ở ngay nhiệt độ phòng.

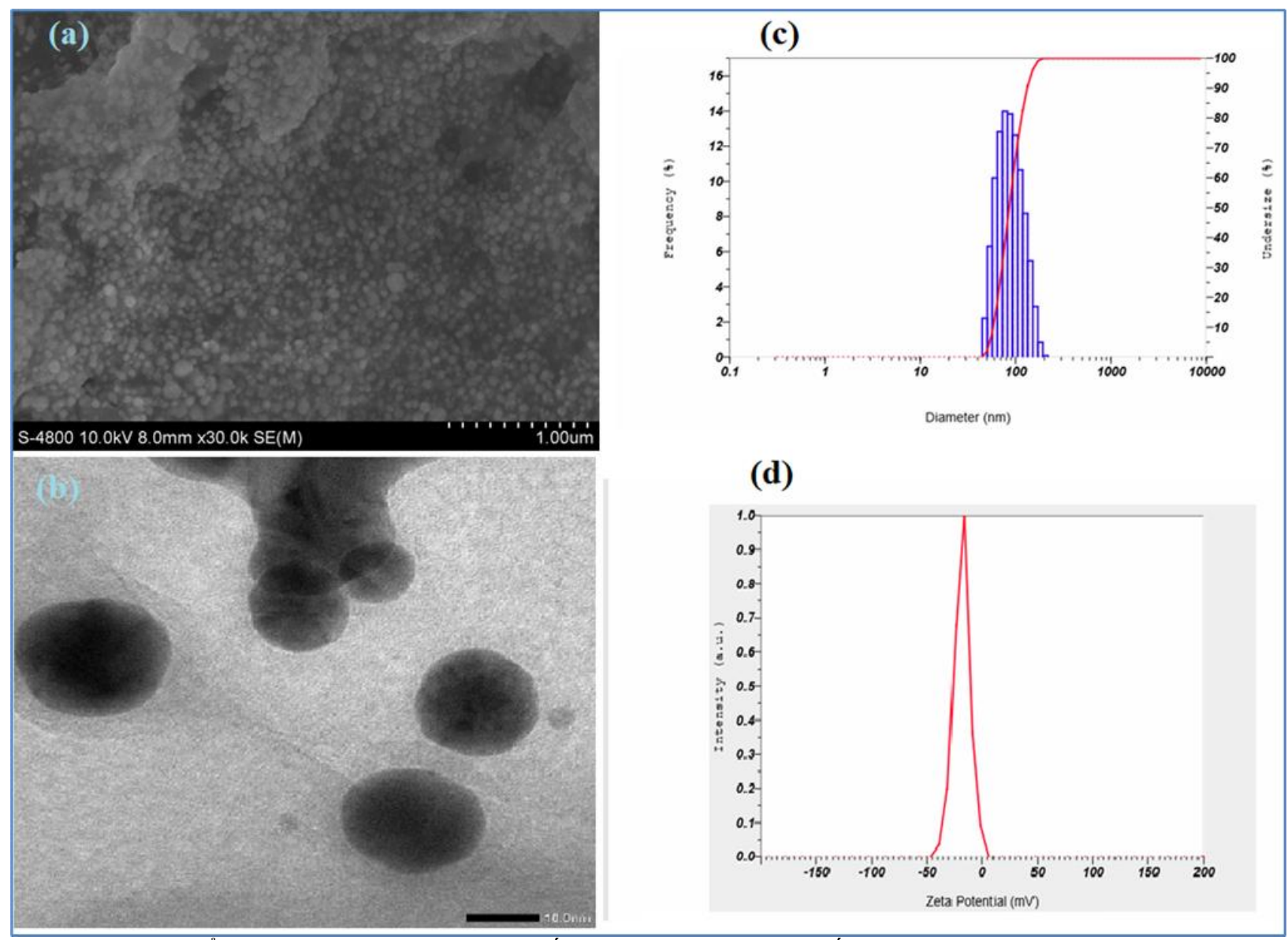

Hình 5: Ảnh SEM (a), TEM (b), phân bố kích thước hạt (c), và thế zeta của dung dịch PP_AuNPs

Phương pháp TG/DTA đã được sử dụng để xác định tính chất nhiệt của mẫu PP_AuNPs (Hình (6)). Mẫu PP_AuNPs sau tổng hợp được mang đi ly tâm để loại bỏ các thành phần vô cơ tan trong nước, sau đó sấy khô. Trong giai đoạn đầu, khối lượng nano vàng giảm gần $6 \%$ tương ứng với đỉnh peak thu nhiệt ở $89^{\circ} \mathrm{C}$ có thể giải thích là do sự bay hơi của các hợp chất hữu cơ và của hơi nước. Giai đoạn sau trọng lượng mẫu PP_AuNPs giảm tiếp $22 \%$ (peak tỏa nhiệt ở $306^{\circ} \mathrm{C}$ ) và cuối cùng giảm $14,1 \%$ (peak tỏa nhiệt $495^{\circ} \mathrm{C}$ ). Sự giảm khối lượng trong giai đoạn sau là do phân hủy nhiệt (sự oxy hóa) của các hợp chất hữu cơ bao bọc bên ngoài nano vàng. Như vậy, lượng vàng trong mẫu PP_AuNPs chiếm $53 \%$. Sự phân hủy nhiệt của các phân tử hữu cơ trên bề mặt của các hạt nano vàng khẳng định rằng các hợp chất hữu cơ có trong dịch chiết từ vỏ trái thơm đóng vai trò quan trọng trong việc khử các ion vàng và ổn định của các hạt nano vàng tạo thành. 


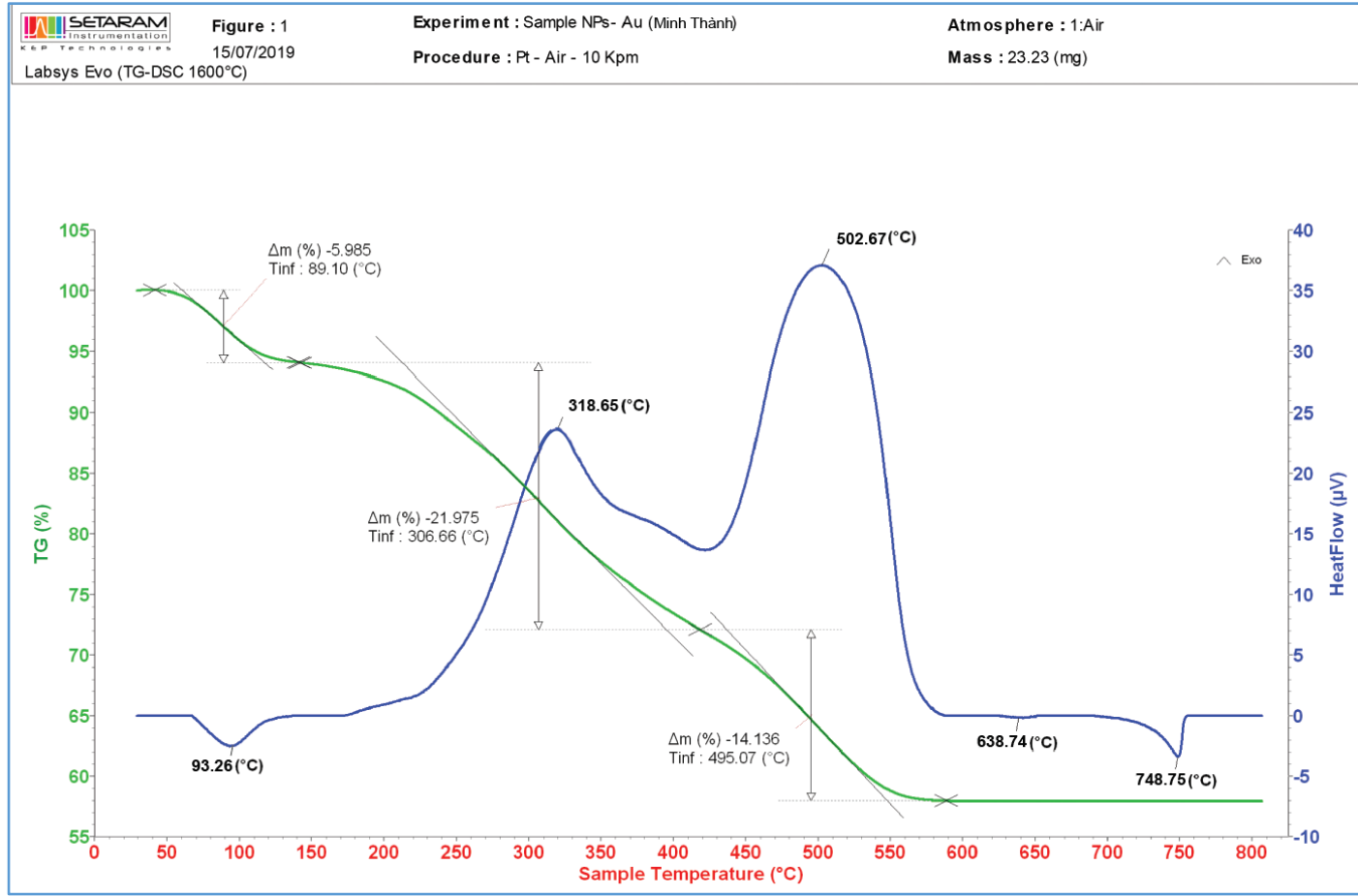

Hình 6: Đường cong TG và TGA của mẫu PP_AuNPs

\subsection{Khả năng xúc tác của PP_AuNPs}

Sau khi thêm dung dịch $\mathrm{NaBH}_{4}$ vào dung dịch 4-NP màu vàng, màu của dung dịch chuyển sang màu vàng đậm và đỉnh cực hấp thụ đại ghi nhận ở bước sóng $400 \mathrm{~nm}$ do sự hình thành ion 4-nitrophenolate trong kiềm trung bình, mặc dù phản ứng phân hủy không xảy ra. Ngay khi bổ sung nano vàng vào hỗn hợp phản ứng, màu của dung dịch dần dần nhạt đi và biến mất. Quang phổ UV-Vis về sự thay đổi về độ hấp thụ quang học của hỗn hợp phản ứng theo thời gian được trình bày ở Hình 7(a). Sự giảm dần của độ hấp thụ ở đỉnh $400 \mathrm{~nm}$ và sự xuất hiện đỉnh mới cao dần ở bước sóng $300 \mathrm{~nm}$ chứng tỏ quá trình phân hủy 4-NP đang xảy ra để tạo thành 4-aminophenol (4-AP). Kết quả cho thấy sự phân hủy 4-NP với xúc tác nano vàng đã được hoàn thành trong 12 phút với bằng chứng từ sự hấp thụ quang học gần như bằng không tại đỉnh 400 nm đặc trưng của 4-NP. Mối quan hệ tuyến tính giữa LnA và thời gian phản ứng đã xác định hằng số tốc độ phản ứng $\mathrm{k}=0,233$ phút $^{-1}$ (Hình 7(b)).

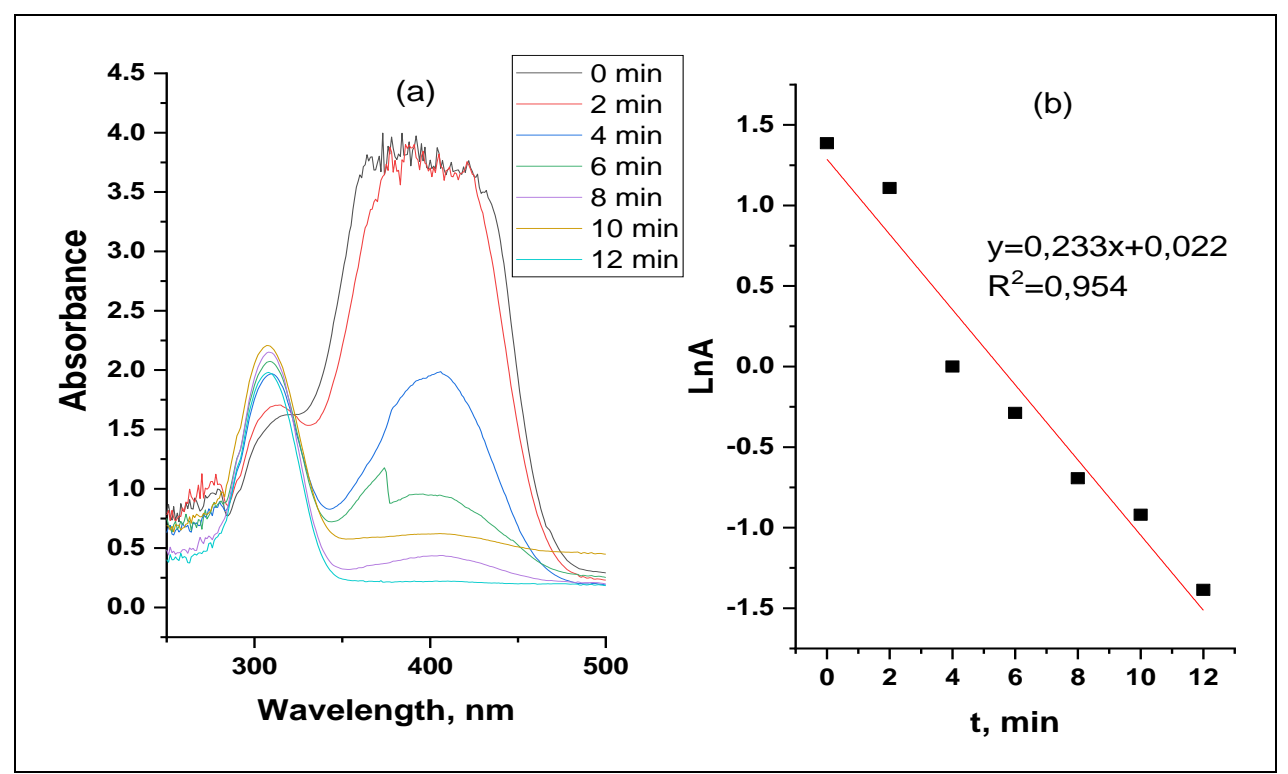

Hình 7: Phổ UV-Vis khảo sát phản ứng xúc tác (a) và đường hồi quy tuyến tính Ln(A) theo thời gian (b) 


\section{KẾT LUẬn}

Tóm lại, các hạt nano vàng hình cầu có kích thước hạt trong khoảng từ 5 đến $15 \mathrm{~nm}$ đã được sinh tổng hợp thành công bằng phương pháp tổng hợp xanh mà không sử dụng bất kỳ chất khử và chất ổn định thương mại đắt tiền nào. Công trình này đã chứng minh hiệu quả của dịch chiết từ vỏ trái thơm - nguồn phế thải nông nghiệp trong việc khử các ion vàng và làm bền các hạt nano vàng tạo thành. Các hạt keo nano vàng phân tán tốt trong môi trường nước và ổn định trong dung dịch hơn một tuần. Các hạt PP_AuNPs thu được cũng thể hiện hoạt tính xúc tác mạnh mẽ đối với phản ứng khử 4-NP. Do đó, các hạt PP_AuNPs sinh tổng hợp có thể được coi là một chất xúc tác hiệu quả thân thiện với môi trường ứng dụng trong xử lý các chất ô nhiễm hữu cơ.

\section{TÀI LIỆU THAM KHẢO}

[1] N. K. R. Bogireddy, U. Pal, L. M. Gomez and V. Agarwal, Size controlled green synthesis of gold nanoparticles using Coffea arabica seed extract and their catalytic performance in 4-nitrophenol reduction, RSC Advances, vol. 8, no. 44, pp. 24819-24826, 2018.

[2] T. T.-N. Nguyen, T.-T. Vo, B. N.-H. Nguyen, D.-T. Nguyen, V.-S. Dang, C.-H. Dang and T.-D. Nguyen, Silver and gold nanoparticles biosynthesized by aqueous extract of burdock root, Arctium lappa as antimicrobial agent and catalyst for degradation of pollutants, Environmental Science and Pollution Research, vol. 25, no. 34, pp. 3424734261, 2018.

[3] T.-T. Vo, C.-H. Dang, V.-D. Doan, V.-S. Dang and T.-D. Nguyen, Biogenic synthesis of silver and sold nanoparticles from Lactuca indica leaf extract and their application in catalytic degradation of toxic compounds, Journal of Inorganic and Organometallic Polymers and Materials, vol. 30, pp. 388-399, 2020.

[4] A. Gangula, R. Podila, M. Ramakrishna, L. Karnam, C. Janardhana and A. M. Rao, Catalytic Reduction of 4Nitrophenol using Biogenic Gold and Silver Nanoparticles Derived from Breynia rhamnoides, Langmuir, vol. 27, no. 24, pp. 15268-15274, 2011.

[5] I. Hussain, N. B. Singh, A. Singh, H. Singh and S. C. Singh, Green synthesis of nanoparticles and its potential application, Biotechnol. Lett., vol. 38, no. 4, pp. 545-560, 2016.

[6] A. Gour and N. K. Jain, Advances in green synthesis of nanoparticles, Artificial Cells, Nanomedicine, Biotechnology, vol. 47, pp. 844-851, 2019.

[7] Y. Guo, N. Jiang, L. Zhang and M. Yin, Green synthesis of gold nanoparticles from Fritillaria cirrhosa and its anti-diabetic activity on Streptozotocin induced rats, Arab. J. Chem., 2020.

[8] S. Balasubramanian, S. M. J. Kala and T. L. Pushparaj, Biogenic synthesis of gold nanoparticles using Jasminum auriculatum leaf extract and their catalytic, antimicrobial and anticancer activities, J. Drug Deliv. Sci. Technol., vol. 57, pp. 101620, 2020.

[9] M. Uzma, N. Sunayana, V. B. Raghavendra, C. S. Madhu, R. Shanmuganathan and K. Brindhadevi, Biogenic synthesis of gold nanoparticles using Commiphora wightii and their cytotoxic effects on breast cancer cell line (MCF7), Process Biochem. 2020.

[10] I. S. Unal, A. Demirbas, I. Onal, N. Ildiz and I. Ocsoy, One step preparation of stable gold nanoparticle using red cabbage extracts under UV light and its catalytic activity, J. Photochem. Photobiol. B Biol., vol. 204, pp. 111800, 2020. 
[11] M. A. Rabeea, M. N. Owaid, A. A. Aziz, M. S. Jameel and M. A. Dheyab, Mycosynthesis of gold nanoparticles using the extract of Flammulina velutipes, Physalacriaceae, and their efficacy for decolorization of methylene blue, $J$. Environ. Chem. Eng., pp. 103841, 2020.

[12] T. Zhang, M. Dang, W. Zhang and X. Lin, Gold nanoparticles synthesized from Euphorbia fischeriana root by green route method alleviates the isoprenaline hydrochloride induced myocardial infarction in rats, J. Photochem. Photobiol. B Biol., vol. 202, pp. 111705, 2020.

[13] C. Vijilvani, M. R. Bindhu, F. C. Frincy, M. S. AlSalhi, S. Sabitha, K. Saravanakumar, S. Devanesan, M. Umadevi, M. J. Aljaafreh and M. Atif, Antimicrobial and catalytic activities of biosynthesized gold, silver and palladium nanoparticles from Solanum nigurum leaves, J. Photochem. Photobiol. B Biol., vol. 202, pp. 111713, 2020. 14. A. Singhal and A. Gupta, Efficient utilization of Sal deoiled seed cake (DOC) as reducing agent in synthesis of silver nanoparticles: Application in treatment of dye containing wastewater and harnessing reusability potential for cost-effectiveness, J. Mol. Liq., vol. 268, pp. 691-699, 2018.

[15] S. Khan, W. Runguo, K. Tahir, Z. Jichuan and L. Zhang, Catalytic reduction of 4-nitrophenol and photo inhibition of Pseudomonas aeruginosa using gold nanoparticles as photocatalyst, J. Photochem. Photobiol. B Biol., vol. 170, pp. 181-187, 2017.

[16] S. S. Hassan, K. Carlson, S. K. Mohanty, Sirajuddin and A. Canlier, Ultra-rapid catalytic degradation of 4 nitrophenol with ionic liquid recoverable and reusable ibuprofen derived silver nanoparticles, Environ. Pollut., vol. 237, pp. 731-739, 2018.

[17] G. Wu, X. Liu, P. Zhou, L. Wang, M. Hegazy, X. Huang and Y. Huang, A facile approach for the reduction of 4-nitrophenol and degradation of congo red using gold nanoparticles or laccase decorated hybrid inorganic nanoparticles/polymer-biomacromolecules vesicles, Mater. Sci. Eng. C, vol. 94, pp. 524-533, 2019.

[18] A. A. Al-Kahtani, T. Almuqati, N. Alhokbany, T. Ahamad, M. Naushad and S. M. Alshehri, A clean approach for the reduction of hazardous 4-nitrophenol using gold nanoparticles decorated multiwalled carbon nanotubes, $J$. Clean. Prod., vol. 191, pp. 429-435, 2018.

[19] T. M.-T. Nguyen, T. T.-T. Huynh, C.-H. Dang, D.-T. Mai, T. T.-N. Nguyen, D.-T. Nguyen, V.-S. Dang, T.-D. Nguyen and T.-D. Nguyen, Novel biogenic silver nanoparticles used for antibacterial effect and catalytic degradation of contaminants, Res. Chem. Intermed., vol. 46, pp. 1975-1990, 2020.

[20] J. C. Buzby, H. F. Wells and J. Hyman, The Estimated Amount, Value, and Calories of Postharvest Food Losses at the Retail and Consumer Levels in the United States, EIB-121, U.S. Department of Agriculture, Economic Research Service: Washington, DC, USA, 2014.

[21] P. R. Ghosh, D. Fawcelt, S. B. Sharma and G. E. J. Poinern, Production of High-Value Nanoparticles via Biogenic Processes Using Aquacultural and Horticultural Food Waste, Materials, vol. 10, no. 8, pp. 852, 2017.

[22] N. Basavegowda, A. Sobczak-Kupiec, D. Malina, H. S. Yathirajan, V. R. Keerthi, N. Chandrashekar, S. Dinkar and L. Padmanabhan, Plant mediated synthesis of gold nanoparticles using fruit extracts of Ananas comosus (L.) (pineapple) and evaluation of biological activities, Adv. Mat. Lett., vol. 4, no. 5, pp. 332-337, 2013.

[23] T. Zhang, M. Dang, W. Zhang, X. Lin, Gold nanoparticles synthesized from Euphorbia fischeriana root by green route method alleviates the isoprenaline hydrochloride induced myocardial infarction in rats, J. Photochem. Photobiol. B Biol., vol. 202, pp. 11170, 2020. 

VỎ TRÁI THƠM XÚC TÁC CHO PHẢN UÚNG XỬ LÝ 4-NITROPHENOL

[24] K. X. Lee, K. Shameli, M. Miyake, N. Kuwano, N. B. B. A. Khairudin, S. E. B. Mohamad and Y. P. Yew, Green synthesis of gold nanoparticles using aqueous extract of Garcinia mangostana fruit peels, J. Nanomater., vol. 2016, pp. 1-7, 2016.

[25] P. K. Francis, S. Sivadasan, A. Avarachan and A. Gopinath, A novel green synthesis of gold nanoparticles using seaweed Lobophora variegata and its potential application in the reduction of nitrophenols, Part. Sci. Technol., vol. 38, pp. 365-370, 2020.

[26] V. D. Doan, V. T. Le, T. D. Nguyen, T. L. H. Nguyen and H. T. Nguyen, Green synthesis of silver nanoparticles using Aganonerion polymorphum leaves extract and evaluation of their antibacterial and catalytic activity, Mater. Res. Express, vol. 6, no. 11, 2019.

[27] V. D. Doan, V. S. Luc, T. L. H. Nguyen, T. D. Nguyen and T. D. Nguyen, Utilizing waste corn-cob in biosynthesis of noble metallic nanoparticles for antibacterial effect and catalytic degradation of contaminants, Environ. Sci. Pollut. Res., vol. 27, pp. 6148-6162, 2020.

Ngày nhận bài: 28/03/2020

Ngày chấp nhận đăng:19/08/2020 